\title{
Better data to drive more effective care for people with latent tuberculosis infection in Canada
}

\author{
Beverley M. Essue PhD, Deborah Milinkovic MA, Stephen Birch PhD
}

Cite as: CMAJ 2018 June 11;190:E700-1. doi: 10.1503/cmaj.180488

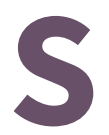

eventy percent of recent migrants to Canada come from areas where tuberculosis (TB) is endemic, and $50 \%$ are estimated to have latent TB infection. ${ }^{1}$ People with latent TB infection in Canada currently number over 1.5 million, ${ }^{2}$ but this could rise by 120000 new cases each year given projected immigration patterns. The estimated lifetime risk of reactivation (active TB developing in a person with latent TB infection) is 5\%-10\%. ${ }^{1}$ Based on current estimates of treatment completion rates for latent TB infection of $16 \%-30 \%, 3,4$ this could translate to an annual increase of 4000 cases of active TB. Although this projection may seem improbably high relative to current rates, the massive data gaps and the ad hoc screening for latent TB infection ${ }^{3-5}$ create challenges for accurate forecasting. However, recent spikes in active TB cases in Ontario ${ }^{5}$ and England, ${ }^{6}$ which have been driven by reactivation of latent TB infection among foreign-born individuals, give cause for concern.

The problem with data is twofold. Canada's immigration medical screening program does not specifically require screening for latent TB infection. In addition, whereas TB is legally reportable in all provinces and territories, latent TB infection is not explicitly identified in most lists of reportable diseases, which leads to inconsistency in the collection and reporting of cases of latent TB infection and relevant risk factors in the Integrated Public Health Information System (iPHIS), the repository of all reportable communicable diseases used for provincial and national surveillance and planning. Consequently, contemporary, population-level data on the characteristics that increase the likelihood of reactivation of latent TB infection are lacking, as well as the total number of individuals in Canada with latent TB infection. A 2015 study found less than $3 \%$ of cases of TB and less than $5 \%$ of cases of latent TB infection among recent immigrants to Canada were identified through the immigration medical screening, ${ }^{8}$ indicating weaknesses in the current systems.

Much focus has been on improving adherence to treatment for latent TB infection. ${ }^{9}$ However, the more pressing question that public health and health services planners should be concerned about is whether we are reaching the right people. The ad hoc nature of screening for latent TB infection means that those with the lowest risk of reactivation (e.g., young, healthy medical students) are more likely to access treatment for latent TB infection, and those who are at the greatest risk (e.g., individuals who are immunocompromised, from low socioeconomic backgrounds or from certain migrant populations) are less likely to be identified and to access treatment. ${ }^{4,10}$

\section{KEY POINTS}

- Canada lacks a uniform and systematic approach to screening, surveillance and treatment for latent tuberculosis (TB) infection, making the current processes ill-equipped to identify and prioritize individuals who are most at risk for reactivation of TB.

- The burden of latent TB infection in Canada is being carried by some of the most disadvantaged groups.

- We have a moral obligation to ensure that those who will benefit most from treatment for latent TB infection are identified, prioritized and supported to initiate and complete treatment to ensure equity in access to care.

- An accurate record of cases of latent TB infection and risk factors is needed to better monitor and act on trends in incidence of latent TB infection and reactivation.

- Improved data quality will also allow public health units to better monitor the effectiveness and impact of their TB control strategies and will support a more equitable and fair use of resources.

A recent Ontario study with latent TB infection stakeholders (i.e., planners, providers and recipients of services) found inconsistencies among providers in their understanding of reporting requirements, screening practices and treatment processes, resulting in marked differences across practices. ${ }^{7}$ A study of surveillance using data from iPHIS found that data on risk factors were missing for more than $50 \%$ of reported cases of latent TB infection ${ }^{9}$ and, where reported, data were inaccurate for more than $50 \%$ of cases. ${ }^{9}$ A recent analysis of data from Ontario found that $30 \%$ of cases were missing information on country of origin and $90 \%$ were missing information on comorbidities. ${ }^{3}$ This unacceptable data quality compromises evidence-informed planning and policy development. The explicit classification of latent TB infection as a reportable condition with mandatory reporting fields, such as country of origin, comorbidities, and other risk factor fields, could help improve data quality. But this comes at a cost as there is a substantial resource burden associated with data collection pertaining to latent TB infection..$^{10}$ There are also challenges with identifying latent TB infection accurately because of a lack of a gold standard test, and there is the potential to cause harm because of the stigma associated with latent TB infection. In spite of these concerns, some US states have made reporting mandatory, 
England is implementing systematic screening and has made the interferon- $\gamma$ release assays (IGRA) blood test freely available, and both the United States and Canada recommend the IGRA test for those previously vaccinated for TB. Yet a qualitative study indicated that the sentiment among some planners is that maintaining the status quo for data collection and reporting is "good enough" to maintain stable TB rates in Ontario, for example. ${ }^{7}$

And herein lies the problem. Although the incidence of TB in Canada is low (4.8 per 100000), within some subgroups the incidence parallels rates found in endemic TB countries. A 2016 analysis showed the incidence of TB among Indigenous Canadians to be 41 times higher than among non-Indigenous Canadians and 170 per 100000 among Inuit people. The incidence among foreign-born individuals is 34.1 per 100000.12 The burden of latent TB infection is being carried by some of the most disadvantaged groups in Canada: the poor, the sick, immigrants and Indigenous people. There is a moral and equity imperative to ensure that those who will benefit most from treatment for latent TB infection are identified, prioritized and supported to initiate and complete treatment to ensure equity in access to care. Efforts focused on improving data capture, data collection, and recording of risk factors of people with latent TB infection will allow public health units to better monitor the effectiveness and impact of their TB control strategies and will support a more equitable and fair use of resources.

Canada lacks a uniform and systematic approach to screening, surveillance and treatment for latent TB infection, making the current processes ill-equipped to identify and prioritize individuals who are most at risk for reactivation of TB. Notwithstanding the challenges of identifying latent TB infection correctly, there is a need for a more systematic approach to surveillance, to ensure routine screening of people who originate from, or regularly travel to, endemic TB countries. This includes better mechanisms for information sharing between federal, provincial and regional levels of authority. Complete, comprehensive data will facilitate better and more equitable care for latent TB infection, better identification and management of risk factors, and support the dissemination of processes that are more effective for initiation and completion of treatment. But it all starts with identification. Without an accurate record of cases of latent TB infection and risk factors, the data will be biased and we will miss opportunities to monitor and act on trends in incidence of latent TB infection and reactivation.

Optimal management of latent TB infection is a key element of TB control in Canada. It is a critical component of the strategy to eliminate TB in northern Canada by 2030 and is critical to achieving the World Health Organization's goal to eradicate TB in low-incidence countries. It is incumbent on Immigration, Refugees and Citizenship Canada (immigration medical screening program), the Public Health Agency of Canada, and the public health agencies and ministries of health in the provinces and territories to better coordinate efforts and information sharing to ensure improvements in data availability, quality and consistency. This, combined with the systematic screening of high-risk populations, will go a long way in enabling Canada to meet this national and global public health priority.

\section{References}

1. Canadian Tuberculosis Standards, 7th edition. Ottawa: Centre for Communicable Diseases and Infection Control, Public Health Agency of Canada; 2014

2. Greenaway C, Sandoe A, Vissandjee B, et al.; Canadian Collaboration for Immigrant and Refugee Health. Tuberculosis: evidence review for newly arriving immigrants and refugees. CMAJ 2011;183:E939-51.

3. Milinkovic D, Birch S, Scott F, et al. A cohort study of factors associated with LTBI treatment adherence in Hamilton, Canada. Can J Infect Control. In press.

4. Rivest P, Street MC, Allard R. Completion rates of treatment for latent tuberculosis infection in Quebec, Canada from 2006 to 2010. Can J Public Health 2013;104: e235-9.

5. TB cases nearly double average yearly rate, Public Health reports. CBC News 2017 Nov. 8. Available: www.cbc.ca/news/canada/kitchener-waterloo/tuberculosis-tb -waterloo-region-rise-more-cases-1.4391211 (accessed 2018 Feb. 25).

6. Collaborative tuberculosis strategy for England: 2015 to 2020. London (UK): Public Health England; 2015.

7. Milinkovic D, Birch S, Scott F, et al. Investigating barriers to latent tuberculosis treatment: a qualitative analysis on the impact of LTBI prioritization on program planning and service delivery. In: Canadian Public Health Association Conference; May 29-31, 2018; Montréal, Que.

8. Khan K, Hirji MM, Miniota J, et al. Domestic impact of tuberculosis screening among new immigrants to Ontario, Canada. CMAJ 2015;187:E473-81.

9. Majerovich JA, Fernandes L, Varia M. Evaluation of latent tuberculosis infection surveillance in Peel region, Ontario, 2010-2014. Can Commun Dis Rep 2017; 43:114-8.

10. Liu Y, Birch S, Newbold KB, et al. The barriers to treatment adherence for individuals with latent tuberculosis infection: a systematic search and narrative synthesis of the literature. Int J Health Plann Manage 2018 Feb. 12 [Epub ahead of print]. doi:10.1002/hpm.2495.

11. de Vries G, van Hest R, Bakker M, et al. Policy and practice of programmatic management of latent tuberculosis infection in The Netherlands. J Clin Tuberc Other Mycobact Dis 2017;7:40-8.

12. Vachon J, Gallant V, Siu W. Tuberculosis in Canada, 2016. Can Commun Dis Rep 2018;44:75-81. Available: www.canada.ca/en/public-health/services/reports -publications/canada-communicable-disease-report-ccdr/monthly-issue/2018-44 /issue-3-4-march-1-2018/article-1-tuberculosis-2016.html (accessed 2018 Feb. 25).

\section{Competing interests: None declared.}

This article has been peer reviewed.

Affiliations: Department of Health Research Methods, Evidence, and Impact (Essue, Milinkovic, Birch), and Centre for Health Economics and Policy Analysis (Essue, Milinkovic), McMaster University, Hamilton, Ont.; School of Public Health (Essue), The University of Sydney, Sydney, Australia; Centre for the Business and Economics of Health (Birch), The University of Queensland, Brisbane, Australia; University of Manchester (Birch), Manchester, UK

Contributors: All of the authors drafted the manuscript, provided critical review and comments, approved the final version to be published and agreed to be accountable for all aspects of the work.

Correspondence to: Beverley Essue, beverley.essue@sydney.edu.au 